\title{
Effects of Intranasal Oxytocin on the Blood Oxygenation Level-Dependent Signal in Food Motivation and Cognitive Control Pathways in Overweight and Obese Men
}

\author{
Franziska Plessow ${ }^{*, 1,2}$, Dean A Marengi', Sylvia K Perry', Julia M Felicione ${ }^{3}$, Rachel Franklin ${ }^{3}$, Tara M Holmes $^{4}$, \\ Laura M Holsen ${ }^{5,6}$, Nikolaos Makris ${ }^{6,7,8}$, Thilo Deckersbach ${ }^{3,6}$ and Elizabeth A Lawson ${ }^{1,2}$ \\ 'Neuroendocrine Unit, Massachusetts General Hospital, Boston, MA, USA; ²Department of Medicine, Harvard Medical School, Boston, MA, USA; \\ ${ }^{3}$ Division of Neurotherapeutics, Massachusetts General Hospital, Charlestown, MA, USA; ${ }^{4}$ Clinical Research Center, Massachusetts General \\ Hospital, Boston, MA, USA; ${ }^{5}$ Division of Women's Health, Department of Medicine and Department of Psychiatry, Brigham and Women's \\ Hospital, Boston, MA, USA; ${ }^{6}$ Department of Psychiatry, Harvard Medical School, Boston, MA, USA; ${ }^{7}$ Athinoula A. Martinos Center for Biomedical \\ Imaging, Charlestown, MA, USA; ${ }^{8}$ Center for Morphometric Analysis, Department of Psychiatry, Massachusetts General Hospital, Boston, MA, USA
}

Recent research indicates that the hypothalamic neuropeptide hormone oxytocin is a key central nervous system factor in the regulation of food intake and weight. However, the mechanisms underlying the anorexigenic effects of oxytocin in humans are unknown and critical to study to consider oxytocin as a neurohormonal weight loss treatment. We performed a randomized, double-blind, placebo-controlled crossover study with single-dose intranasal oxytocin $(24 \mathrm{U})$ in ten overweight or obese, otherwise healthy men. Following oxytocin/ placebo administration, participants completed an established functional magnetic resonance imaging food motivation paradigm. We hypothesized that oxytocin would reduce the blood oxygenation level-dependent (BOLD) signal to high-calorie food vs non-food visual stimuli in the ventral tegmental area (VTA), the origin of the mesolimbic dopaminergic reward system. Following oxytocin administration, compared to placebo, participants showed bilateral VTA hypoactivation to high-calorie food stimuli. A secondary exploratory whole-brain analysis revealed hypoactivation in additional hedonic (orbitofrontal cortex, insula, globus pallidus, putamen, hippocampus, and amygdala) and homeostatic (hypothalamus) food motivation and hyperactivation in cognitive control (anterior cingulate and frontopolar cortex) brain regions following oxytocin administration vs placebo. Oxytocin administration reduces the BOLD signal in reward-related food motivation brain regions, providing a potential neurobiological mechanism for the anorexigenic oxytocin effects in humans. Furthermore, our data indicate that oxytocin administration reduces activation in homeostatic and increases activation in cognitive control brain regions critically involved in regulating food intake and resolving affective conflict, respectively. Future studies are required to link these changes in brain activation to oxytocin effects on food intake and weight.

Neuropsychopharmacology (2018) 43, 638-645; doi:I0.1038/npp.2017.226; published online I8 October 2017

\section{INTRODUCTION}

Oxytocin is currently under investigation as a potential novel neurohormonal treatment for obesity (Blevins and Baskin, 2015). Obesity represents a major public health concern in need of more effective and tolerable treatments. In the United States, $69 \%$ of adults are overweight with 35\% meeting the criteria for obesity, an epidemic that is associated with diabetes mellitus and increased mortality (Ogden et al, 2014). Even though complications resulting from obesity may be reversible with weight reduction, meaningful weight loss is difficult to achieve and maintain with available medical treatments. In addition, current

* Correspondence: Dr F Plessow, Neuroendocrine Unit, Massachusetts General Hospital, 55 Fruit Street BUL457B, Boston, MA 02114, USA. Tel: + I-6I7-726-0784; Fax: + |-6|7-726-5072;

E-mail: fplessow@mgh.harvard.edu

Received 7 July 2017; revised 29 August 2017; accepted 15 September 2017; accepted article preview online 20 September 2017 treatment options are associated with frequent complications (bariatric surgery; Gloy et al, 2013) and significant side effects (FDA-approved drugs; Yanovski and Yanovski, 2014). The nonapeptide hormone oxytocin is primarily produced in the paraventricular and supraoptic nuclei of the hypothalamus and secreted throughout the brain and to the peripheral circulation via the posterior pituitary. Repeated oxytocin administration has been shown to induce significant weight loss in rodent, primate, and human obesity (Blevins and Baskin, 2015; Blevins et al, 2015; Zhang et al, 2013). Importantly, short-term administration in humans has been associated with minimal side effects (Anagnostou et al, 2012; Zhang et al, 2013). In animal models, the beneficial effects of oxytocin on weight are due to multiple processes, including increased energy expenditure and lipolysis and reduced food intake (Blevins and Baskin, 2015). For example, in fasting rats, central and peripheral oxytocin administration resulted in a dose-dependent reduction in caloric intake and an increased delay before eating (Arletti et al, 1989; Olson et al, 
1991). This effect was reversed by administering an oxytocin antagonist prior to oxytocin exposure, suggesting that this effect is mediated by oxytocin receptors (Arletti et al, 1989).

Recent translational studies using intranasal oxytocin have demonstrated that oxytocin reduces caloric consumption, particularly of palatable foods, without affecting subjective appetite in men (Lawson et al, 2015; Ott et al, 2013; Thienel et al, 2016). However, the mechanisms driving the effect of oxytocin on food intake in humans are unknown, and an understanding of these mechanisms is critical for the development of oxytocin-based therapies for obesity management.

Preclinical data indicate that oxytocin may reduce food intake in part by modulating activation of hedonic food motivation pathways. Although homeostatic modulation of food intake determining hunger and appetite is largely controlled by the hypothalamus, which receives and integrates peripheral anorexigenic and orexigenic signals, hedonic food motivation arises from a widespread network of brain regions known to be involved in reward processing. The ventral tegmental area (VTA), amygdala, insula, basal ganglia (eg, nucleus accumbens), hippocampus, and orbitofrontal cortex (OFC) are associated with hedonic regulation of human appetite, as shown by their selective functional magnetic resonance imaging (fMRI) activation to palatable food stimuli (Holsen et al, 2012; LaBar et al, 2001). At the core of this mesolimbic dopaminergic reward system, the VTA is the origin of its dopaminergic cell bodies and features oxytocin receptors (Loup et al, 1991). In addition, oxytocin neurons project from the paraventricular and supraoptic nuclei of the hypothalamus to the VTA (Sofroniew, 1983). In preclinical studies, oxytocin availability in the VTA has been shown to alter dopamine levels in brain regions downstream from the VTA (eg, in the nucleus accumbens; Melis et al, 2007), providing a potential biological link between oxytocin and motivational behavior including hedonic food intake. In rats, administration of oxytocin into the VTA and nucleus accumbens reduced sucrose intake, whereas these effects were attenuated when an oxytocin receptor blocker was given prior to oxytocin treatment (Herisson et al, 2016; Mullis et al, 2013). In humans, obesity is characterized by fMRI hyperactivation of food motivation brain regions associated with hedonic aspects of appetite and food intake (De Silva et al, 2012; Dimitropoulos et al, 2012).

Although human studies provide evidence that single-dose intranasal oxytocin administration alters the blood oxygenation level-dependent (BOLD) signal to social and emotional stimuli (Domes et al, 2007; Wigton et al, 2015) and monetary rewards (Mickey et al, 2016; Nawijn et al, 2016), to our knowledge, the impact of oxytocin on the spontaneous BOLD signal in reward-related food motivation pathways in response to viewing palatable food images has not yet been examined. We therefore performed a randomized, doubleblind, placebo-controlled crossover pilot study of 24 IU intranasal oxytocin in overweight and obese, otherwise healthy men to investigate oxytocin effects on the BOLD signal in hedonic food motivation brain regions using a wellestablished visual food stimuli fMRI paradigm (Holsen et al, 2012). We hypothesized that when participants view highcalorie foods compared to objects, the BOLD signal in the VTA as a key node for hedonic food motivation would be reduced following oxytocin administration compared to placebo. As the hedonic regulation of appetite emerges from a network of food motivation regions extending beyond the VTA and given that other non-reward functions (eg, homeostasis, cognitive control; Calvo et al, 2014) and their according brain regions (eg, hypothalamus for homeostasis and dorsolateral prefrontal cortex (DLPFC), anterior cingulate cortex (ACC), and frontopolar cortex for cognitive control; Niendam et al, 2012; Saper et al, 2002) are likely to be involved in determining food intake, we performed whole-brain fMRI and, as a secondary exploratory aim, examined the effects of oxytocin on the BOLD signal across the entire brain.

\section{MATERIALS AND METHODS}

\section{Participants}

Ten overweight or obese men, recruited from the community, took part in the study. Inclusion criteria were an age between 18 and 45 years, a body mass index (BMI) between 25 and $40 \mathrm{~kg} / \mathrm{m}^{2}$, regular breakfast eating ( $\geqslant 4$ times per week), and stable weight over the past 3 months. Exclusion criteria were a psychiatric diagnosis, psychotropic medication, a history of eating disorder by Structured Clinical Interview for DSM Disorders-IV (SCID-IV), excessive exercise (running $>25$ miles or exercising $>10 \mathrm{~h}$ in any 1 week) within the last 3 months, active substance abuse, smoking, a history of cardiovascular disease, diabetes mellitus, or gastrointestinal tract surgery, hematocrit below the normal range, untreated thyroid disease, and contraindication for MRI.

\section{Procedures}

This study was approved by the Partners HealthCare Institutional Review Board and conducted in accordance with the Declaration of Helsinki. Following the International Committee of Medical Journal Editors' registration requirement, this clinical trial was preregistered at clinicaltrials.gov (registration number: NCT02276677). All participants provided their written informed consent prior to participation.

Participants were admitted to the Massachusetts General Hospital Clinical Research Center for outpatient screening and two main study visits. At screening, medical history, physical examination (including height and weight measurements), and a blood draw were performed to confirm eligibility. Exercise pattern was assessed by medical history and Paffenbarger Physical Activity Scale (Paffenbarger et al, 1993). In addition, behavior related to food intake was assessed with the Three-Factor Eating Questionnaire (TFEQ), a 51-item self-report questionnaire that provides scores for three factors determining eating behavior, ie, cognitive restraint of eating, disinhibition, and hunger (Stunkard and Messick, 1985). The first main study visit was completed between 6 and 49 days after the screening visit. Screening visits were conducted between November 2014 and May 2015. Main study visits were completed between December 2014 and May 2015.

Main study visits took place in the morning between 6 and 24 days apart. Participants completed a food diary for the $72 \mathrm{~h}$ leading up to each main study visit. In addition, they were asked to fast for at least $10 \mathrm{~h}$ and avoid strenuous exercise and alcohol consumption in the $24 \mathrm{~h}$ prior 
to study visits. Each main study visit started with an update of the medical history and collection of the completed food diary. At visit 1, a copy of the food diary from the $72 \mathrm{~h}$ leading up to the visit was provided to the participant who was instructed to replicate the recorded eating pattern as closely as possible prior to presenting for visit 2 . From the food diaries, a dietician analyzed the nutritional content of the food intake (total caloric intake as well as carbohydrate, fat, and protein content) in the $72 \mathrm{~h}$ prior to both main study visits for each participant using ProNutra (Viocare, Princeton, NJ, USA) to confirm similar conditions preceding oxytocin and placebo visits. In addition, self-reported duration of sleep during the night prior to each main study visit was documented. At 0730 hours, intranasal oxytocin (24 IU, Syntocinon, Novartis, Switzerland, provided by Victoria Pharmacy Zürich, Switzerland) or placebo (same inactive ingredients and packaging, Victoria Pharmacy) nasal spray ( $5 \mathrm{ml}$ bottles with $40 \mathrm{IU} / \mathrm{ml}$ oxytocin or placebo and a $0.1 \mathrm{ml}$ dosage pump) was self-administered with three sprays per nostril under the supervision of a nurse practitioner. For this randomized, double-blind, placebo-controlled crossover study, the research pharmacy randomized the participants 1:1 to one of two drug orders, ie, oxytocin-placebo or placebo-oxytocin. All study personnel and participants were blinded to the randomization. Sixty minutes after oxytocin or placebo administration, participants underwent fMRI. Immediately prior to as well as 30 and $90 \mathrm{~min}$ following oxytocin/placebo administration, appetite was assessed on the dimensions Hunger and Desire to Eat Favorite Food on visual analog scales of 10-cm length, anchored by 'not at all' on the left and 'extremely' on the right. At the same time points, current levels of anxiety were measured using the state scale of the State-Trait Anxiety Inventory (STAI; Spielberger et al, 1983). For 20 statements (eg, 'I feel nervous.'), participants were asked to indicate how much the statement matched their current feelings on a four-point Likert-like scale ranging from 1 ('not at all') to 4 ('very much so').

\section{Functional MRI Paradigm}

Functional MRI scanning was performed during a wellestablished food motivation paradigm that has been reported in detail elsewhere (Holsen et al, 2012). In short, participants viewed 100 high-calorie food stimuli (50 savory, 50 sweet items), 100 low-calorie food stimuli, 100 household objects, and 100 fixation stimuli in a block design. Each stimulus was presented once for $3 \mathrm{~s}$ using Presentation software (Neurobehavioral Systems, Albany, CA, USA). Participants were instructed to press a button when pictures changed to insure their attention to stimuli. A total of five 4-min runs with five images in each block and 16 blocks in each run were completed.

\section{MRI Acquisition Parameters}

MRI data were acquired using a Siemens 3T Trio scanner (Siemens, Erlangen, Germany) at the Athinoula A. Martinos Center for Biomedical Imaging. Head movements were restricted with foam cushions. Whole-brain functional imaging was performed using a gradient-echo EPI pulse sequence (33 contiguous oblique-axial slices, 4-mm thick,
$\mathrm{TR} / \mathrm{TE}=2000 / 30 \mathrm{~ms}$, flip angle $=90^{\circ}, \mathrm{FOV}=200 \times 200 \mathrm{~mm}$, 120 total images per run).

\section{Data Analysis}

Comparisons of food intake, sleep duration, appetite, and anxiety between oxytocin and placebo visits were analyzed with Wilcoxon signed-rank tests using JMP Pro (version 13; SAS Institute, Cary, NC, USA).

Functional MRI data were processed using Statistical Parametric Mapping 8 (SPM8; Wellcome Department of Cognitive Neurology, London, UK; www.fil.ion.ucl.ac.uk/spm). Functional MRI images were slice-time corrected, realigned, spatially normalized, and registered to the Montreal Neurological Institute (MNI; www.bic.mni.mcgill.ca) T1 canonical template. Data were then resampled to $2 \mathrm{~mm}^{3}$ voxels and smoothed with a 6-mm isotropic full width at half-maximum Gaussian function. All collected data showed minimal head motion $(<3 \mathrm{~mm}$ linear movement in the orthogonal planes and $<0.5^{\circ}$ radians of angular movement). For each participant, condition effects were estimated at each voxel, and statistical parametric maps (con images) were produced for the high-calorie food and non-food object conditions for both the oxytocin and placebo conditions.

As the primary region of interest (ie, VTA) is very small, preprocessing of oxytocin and placebo conditions was done within the same pipeline to ensure proper alignment of individual scans. These individual con images were then entered into a second-level analysis using a linear flexible factorial model with the factors participant and condition (oxytocin high-calorie stimuli, oxytocin non-food objects, placebo high-calorie stimuli, and placebo non-food objects). Oxytocin-related changes were tested using contrasts that reflected the difference in beta weights between oxytocin high-calorie food images minus oxytocin non-food objects $v s$ placebo high-calorie food images minus placebo non-food objects. For the primary confirmatory analysis of oxytocin effects on VTA activation, we used max voxel coordinates from the left and right hemispheres of the group level statistical maps at the centers of $2.5 \mathrm{~mm}$ spheres and the beta values using Marsbar software. The location of the VTA was verified by a senior neuroanatomist (NM) guided by MRIvisible anatomical landmarks and brain atlases (Parent, 1996; Paxinos and Huang, 1995).

Following the primary analysis, we conducted an exploratory whole-brain analysis (oxytocin high-calorie food images minus oxytocin non-food objects vs placebo high-calorie food images minus placebo non-food objects) with a group level statistical map threshold of $p<0.05$ (uncorrected) with the additional requirement that at least four contiguous voxels exceeded this statistical level. Cohen's $d$ is reported as effect size measure calculated from the mean $t$-statistic for each cluster.

\section{RESULTS}

\section{Participant Characteristics}

Participant characteristics at the screening visit are described in Table 1. Participants were between 23 and 43 years old, and their BMI ranged from 25.3 to $33.7 \mathrm{~kg} / \mathrm{m}^{2}$. They reported minimal to moderate cognitive restraint of eating, 
Table I Participant Characteristics

\begin{tabular}{lcl}
\hline & Mean \pm SEM & Range \\
\hline Age (years) & $31.4 \pm 1.8$ & $23-43$ \\
Body mass index $\left(\mathrm{kg} / \mathrm{m}^{2}\right)$ & $28.9 \pm 0.8$ & $25.3-33.7$ \\
TFEQ $^{a}$ & & \\
Cognitive restraint of eating (range: 0-21) & $8.8 \pm 1.6$ & $1-14$ \\
Disinhibition (range: 0-16) & $3.4 \pm 0.6$ & $1-7$ \\
Hunger (range: 0-14) & $2.3 \pm 0.3$ & $1-3$ \\
\hline
\end{tabular}

Abbreviation: TFEQ, Three-Factor Eating Questionnaire.

$a_{n}=9$.

Table 2 Food Intake, Sleep Duration, Appetite, and Anxiety Reported at Oxytocin and Placebo Visits

\begin{tabular}{|c|c|c|c|}
\hline & Oxytocin & Placebo & $p$ \\
\hline \multicolumn{4}{|c|}{ Food intake per $24 \mathrm{~h}$ in the $72 \mathrm{~h}$ prior to the study visit } \\
\hline Total calories (kcal) & $2,393 \pm 306$ & $2,208 \pm 337$ & 0.359 \\
\hline Carbohydrates (g) & $306 \pm 45$ & $272 \pm 44$ & 0.074 \\
\hline Fat $(\mathrm{g})$ & $85 \pm 11$ & $78 \pm 14$ & 0.496 \\
\hline Protein (g) & $98 \pm 11$ & $98 \pm 18$ & 0.652 \\
\hline Sleep duration previous night ( $h$ ) & $5.7 \pm 0.5$ & $5.4 \pm 0.5$ & 0.438 \\
\hline \multicolumn{4}{|l|}{ Appetite } \\
\hline \multicolumn{4}{|l|}{ Hunger } \\
\hline Prior to drug administration & $4.1 \pm 0.5$ & $4.5 \pm 0.8$ & 0.910 \\
\hline$+30 \min ^{\mathrm{a}}$ & $4.7 \pm 0.8$ & $4.4 \pm 0.9$ & 0.684 \\
\hline$+90 \min ^{\mathrm{a}}$ & $5.3 \pm 1.0$ & $5.9 \pm 0.9$ & 0.375 \\
\hline \multicolumn{4}{|l|}{ Desire to eat favorite food } \\
\hline Prior to drug administration ${ }^{a}$ & $4.5 \pm 0.4$ & $4.1 \pm 0.8$ & 0.910 \\
\hline$+30 \min ^{\mathrm{a}}$ & $4.6 \pm 0.8$ & $4.6 \pm 0.9$ & 1.000 \\
\hline$+90 \min ^{\mathrm{a}}$ & $5.2 \pm 0.9$ & $5.9 \pm 0.9$ & 0.234 \\
\hline \multicolumn{4}{|l|}{ Anxiety } \\
\hline \multicolumn{4}{|l|}{ STAl state } \\
\hline Prior to drug administration & $25.2 \pm 2.5$ & $26.7 \pm 2.3$ & 0.328 \\
\hline$+30 \min ^{\mathrm{a}}$ & $25.3 \pm 2.6$ & $26.4 \pm 3.0$ & 0.250 \\
\hline +90 $\min ^{\mathrm{a}}$ & $26.1 \pm 3.1$ & $25.7 \pm 2.8$ & 0.750 \\
\hline
\end{tabular}

Abbreviation: STAI, State-Trait Anxiety Inventory.

$a_{n}=9$.

Data are reported as mean $\pm \mathrm{SEM}$.

disinhibition, and hunger in their everyday life as indicated by the TFEQ (Table 1). As intended per study design, food intake (ie, total caloric intake and macronutrient content) during the $72 \mathrm{~h}$ leading up to the visits did not differ between oxytocin and placebo visits (Table 2). There were also no differences in sleep duration leading up to the visits. Furthermore, no differences in appetite or anxiety levels were observed between oxytocin and placebo visits (Table 2).

\section{VTA Activation Following Oxytocin Administration vs Placebo}

Following oxytocin administration, compared to placebo, participants showed hypoactivation in the left VTA
$(-6-13-14)$ in response to viewing high-calorie food stimuli compared with the objects, $p=0.004, d=0.91$. We observed a matching hypoactivation in the right VTA $(6-19-17), p=0.015, d=0.73$ (Figure 1).

\section{Whole-Brain Analysis Following Oxytocin Administration vs Placebo}

Whole-brain analysis contrasting high-calorie food stimuli with non-food objects showed that oxytocin reduced fMRI activation in additional reward-related food motivation brain regions, including the medial frontal gyrus (Broadmann areas (BAs) 8-11, 45, and 46), precentral gyrus (BA 6), sulcus callosomarginalis, posterior cingulate gyrus (BA 31), insula, globus pallidus, caudal putamen, thalamus, hippocampus, and amygdala (Table 3). A particularly large hypoactivation following oxytocin administration $v s$ placebo was observed in BAs $9 / 45$ of the medial frontal gyrus, comprising a cluster of activation of 1551 voxels at the $p<0.05$ (uncorrected) threshold. In addition, oxytocin ( $v s$ placebo) reduced activation in the hypothalamus, a brain area central to homeostatic control of feeding. In contrast, oxytocin increased fMRI activation in brain regions involved in cognitive control, ie, the superior frontal gyrus (BA 10) and ACC (BA 32; Table 3).

\section{DISCUSSION}

Although translational studies with intranasal oxytocin have recently demonstrated that oxytocin reduces food intake in men across the weight spectrum (Lawson et al, 2015; Ott et al, 2013; Thienel et al, 2016), the mechanisms driving these anorexigenic effects of oxytocin in humans are unknown. We tested the impact of single-dose intranasal oxytocin (24 IU) on the BOLD signal in hedonic food motivation pathways while participants viewed high-calorie food vs non-food stimuli. We hypothesized that the BOLD signal in the VTA as the origin of the mesolimbic dopaminergic reward system would be reduced after oxytocin administration compared to placebo. Confirming our hypothesis, we found that following oxytocin administration, overweight and obese men showed bilateral hypoactivation in the VTA when viewing highcalorie food stimuli compared to objects. To our knowledge, this is the first report of reduced VTA activation to palatable food stimuli after oxytocin administration.

It is possible that the attenuating effect of oxytocin on the VTA BOLD signal to rewarding stimuli might be of a more general nature. Two studies have previously shown evidence for oxytocin effects on the VTA BOLD signal in foodunrelated contexts and populations different from the overweight and obese men investigated in this study. Gregory et al (2015) showed an increased VTA BOLD response to both crying infant and sexual stimuli in postpartum and nulliparous women after a single dose of $24 \mathrm{IU}$ intranasal oxytocin (the same dose as used in this study, investigating VTA responses to arousing rather than reward stimuli). Mickey et al (2016) showed an altered time course of VTA activation to monetary reward stimuli following intranasal oxytocin administration compared to placebo in healthy men. These findings together with our result of VTA hypoactivation to high-calorie food stimuli 
a
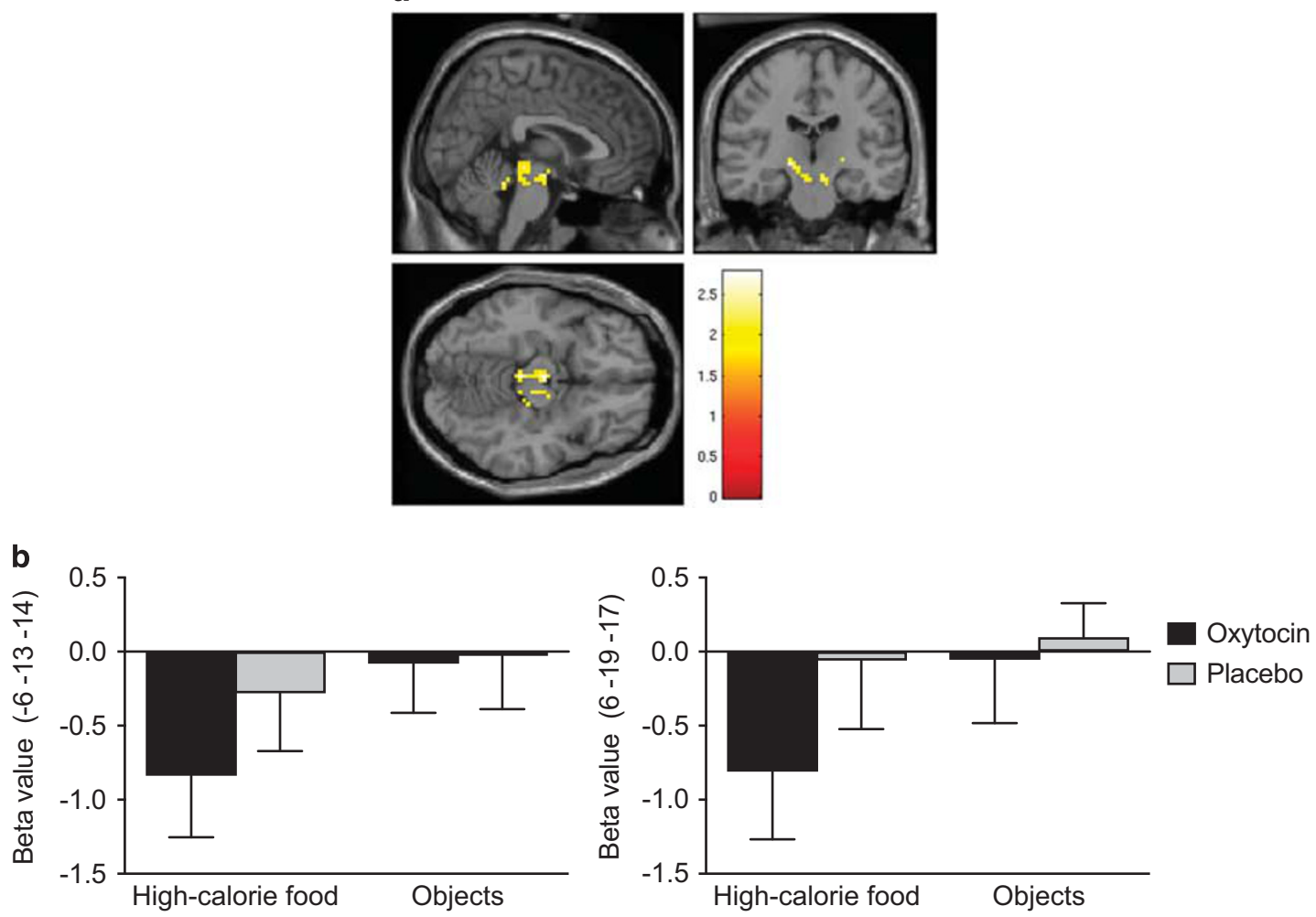

Figure I (a) Activation in the ventral tegmental area (VTA) as a function of condition (oxytocin high-calorie stimuli, oxytocin non-food objects, placebo high-calorie stimuli, and placebo non-food objects; primary confirmatory analysis). Beta values represent differences from the condition means. (b) Beta values for left and right VTA for oxytocin high-calorie stimuli, oxytocin non-food objects, placebo high-calorie stimuli, and placebo non-food objects. Error bars represent SEM. $p<0.05$.

following oxytocin raise novel questions of oxytocin use in conditions characterized by altered reward sensitivity (eg, substance abuse). In the context of human obesity, presenting high-calorie food stimuli induces hyperactivation of hedonic food motivation pathways as compared to normal-weight controls (De Silva et al, 2012; Dimitropoulos et al, 2012). A recent systematic review provides evidence that obese individuals show increased reward sensitivity not only to food but also to monetary rewards (Stojek and MacKillop, 2017). This points toward a generally increased reward sensitivity in this population, and the attenuating effect of oxytocin on the VTA BOLD signal might thus represent a promising counteracting intervention.

A secondary exploratory analysis assessing oxytocin effects on the BOLD signal across the entire brain complemented the finding of VTA hypoactivation in response to high-calorie stimuli following oxytocin administration with hypoactivation of other key brain areas involved in hedonic food motivation. In addition to the VTA, hedonic food motivation pathways comprise the OFC, insula, parts of the basal ganglia, hippocampus, and amygdala (Holsen et al, 2012; LaBar et al, 2001). In response to oxytocin, we found hypoactivation in the medial frontal gyrus including the OFC (BA 11), insula, basal ganglia (globus pallidus, putamen), hippocampus, and amygdala, suggestive of widespread oxytocin effects on the hedonic food motivation neurocircuitry.

Furthermore, the whole-brain analysis revealed hypoactivation in the hypothalamus, the core brain region associated with homeostatic food motivation (Saper et al, 2002), following oxytocin compared to placebo. This is consistent with preclinical data showing that the human ventromedial hypothalamus, representing a brain region that is linked to the control of food intake, is rich in oxytocin receptors (Boccia et al, 2013; Loup et al, 1991). Moreover, a recent study in healthy, normal-weight individuals showed a reduction of hypothalamic activation to visual food stimuli following a single-dose administration of 24 IU intranasal oxytocin (van der Klaauw et al, 2017). Our study extends this finding by providing first evidence that intranasal oxytocin similarly impacts hypothalamus activation in overweight and obese men, where it could serve as a potential weight loss treatment. These data suggest that oxytocin effects on food motivation might not be limited to hedonic food motivation but also extend to homeostatic food regulation. Investigating the contribution of both food motivation pathways to the anorexigenic effects of oxytocin represents an interesting scope for future research.

Finally, we observed effects of oxytocin on regions involved in cognitive control, the processes that enable individuals to suppress behavioral impulses to achieve an internal goal (Miller and Cohen, 2001). The ability to exert control over behavior emerges from a complex neural network centered around the DLPFC (BAs 9 and 46), ACC (BA 32), and frontopolar cortex (BA 10) with connections to other cortical and subcortical (eg, putamen, globus pallidus) brain regions (Niendam et al, 2012). Our exploratory analysis revealed increased activation in the ACC (BA 32) and frontopolar cortex (BA 10)-brain regions that can be linked to cognitive control-following oxytocin administration compared to placebo, whereas no other hyperactivations 
Table 3 Activation in Food Motivation and Cognitive Control Brain Regions to High-Calorie Food Stimuli Compared to Non-Food Objects (Secondary Exploratory Whole-Brain Analysis)

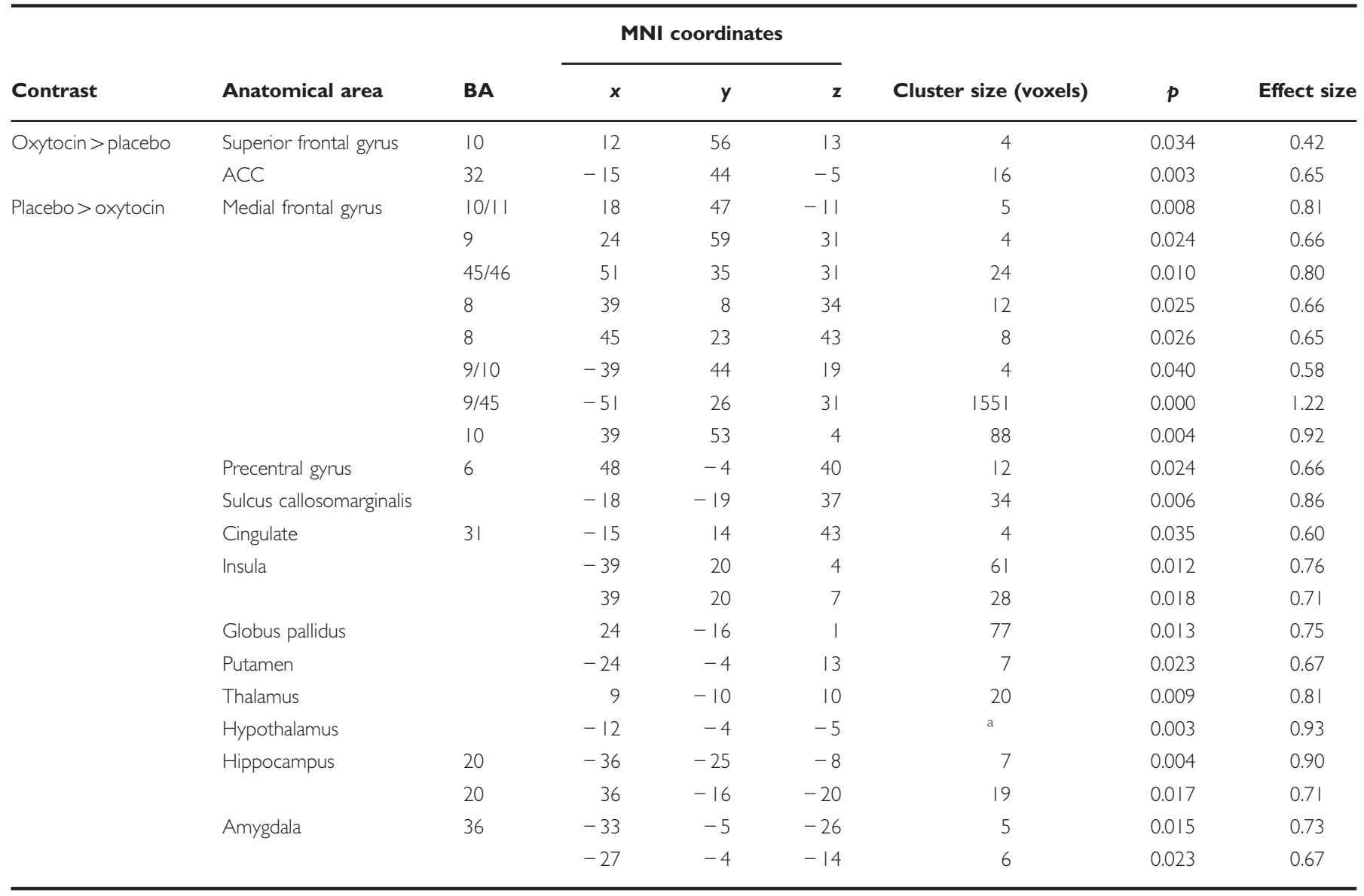

Abbreviations: ACC, anterior cingulate cortex; BA, Broadmann area.

a Voxel size not given (subcluster).

The group level statistical map was thresholded at $p<0.05$ (uncorrected) with the additional requirement of $\geq 4$ voxels in a cluster.

were observed across the entire brain. Cognitive control is assumed to mediate food intake in overweight and obese individuals, as overweight and obese individuals show reduced cognitive control compared with the normal-weight individuals (Nederkoorn et al, 2006; Sellaro and Colzato, 2017), and lower cognitive control has been associated with higher levels of uncontrolled eating and increased food intake in this population (eg, Calvo et al, 2014).

Oxytocin receptors are evident in key cognitive control regions (Skuse and Gallagher, 2009), and oxytocin administration has been demonstrated to promote cortical control over subcortical brain structures by increasing connectivity between neocortical and limbic structures and decreasing limbic-midbrain communication (Domes et al, 2007; Riem et al, 2012). Indeed, a recent study in healthy women showed a trend for an effect of oxytocin in reducing food craving when instructions of actively suppressing food cravings were given (Striepens et al, 2016). This finding was accompanied by an increased BOLD signal in cognitive control brain areas following administration of 24 IU intranasal oxytocin compared to placebo. Our study shows evidence of an oxytocin-cognitive control link in overweight and obese individuals. Furthermore, our study fundamentally differs from Striepens et al (2016) by demonstrating that oxytocin increased activation in cognitive control brain regions even when no suppression of food cravings was required (as no actual food was present) or requested (eg, via instructions to suppress arising food cravings). We speculate that activation of cognitive control brain regions in our paradigm results from a priming (ie, automatic activation) of cognitive control by images of palatable foods. For overweight and obese individuals, palatable food stimuli have a high reward value, driving increased intake of those foods (De Silva et al, 2012; Dimitropoulos et al, 2012). At the same time, (over-) consumption of high-calorie food contributes to maintaining and increasing overweight in those individuals. Thus, in this population, palatable foods represent stimuli with conflicting connotation (approach and avoidance) and action implications attached to them. To abstain from (over-)eating available palatable foods, the desire for consuming them may require counteraction with an increase in cognitive control. Repeated experience of this scenario might eventually result in a welllearned link between palatable foods as aversive signals and increased control demands. This would aid establishing an automatic activation of the cognitive control network in response to palatable food stimuli (Botvinick, 2007; Dreisbach and Fischer, 2012), and oxytocin appears to amplify this automatic recruitment of the cognitive control neurocircuitry. This idea is also supported by the fact that the BOLD signal in the ACC (BA 32), an area that detects and signals the need for 
cognitive control (Mansouri et al, 2009), is amplified following oxytocin administration $v s$ placebo. More specifically, the area of hyperactivation within the (rostral) ACC following oxytocin administration $v s$ placebo $\left(\begin{array}{lll}-15 & 44 & -5\end{array}\right)$ in this crossover study has been implicated in the detection and resolution of affective conflict (Whalen et al, 1998). Future research is required to investigate the potential role of this hyperactivation in cognitive control brain regions in the observed hypoactivation in food motivation pathways following oxytocin administration compared to placebo.

This is the first pilot study investigating the effects of oxytocin on the BOLD signal in response to high-calorie food images in overweight and obese men, and the small sample size represents a limitation of this investigation. Future studies are required to replicate our finding of bilateral VTA hypoactivation to palatable food $v s$ non-food images following oxytocin administration with larger sample sizes. This study focused on men due to the fluctuations in levels of estrogen and progesterone, hormones that regulate oxytocin secretion and receptor distribution (Maldjian et al, 2003), in women. However, it will be important to also study women in the future due to gender specificity of oxytocin effects (Gao et al, 2016). The results of the secondary exploratory whole-brain analysis allow for generating clear hypotheses for future confirmatory studies of oxytocin effects on food motivation and cognitive control pathways in human obesity. Those investigations should also test whether the observed changes of the BOLD signal in food motivation and cognitive control brain regions are accompanied by meaningful behavioral changes. Some brain regions, such as the putamen and insula, have a role in both reward processing and cognitive control. Thus, future studies are needed to understand the functionality of the observed activity changes, for example, by analyzing fMRI during instructed engagement or disengagement of cognitive control and by using functional connectivity analysis approaches.

It can be summarized that key regions of cognitive control are hyperactivated following oxytocin compared to placebo in overweight and obese men, whereas regions of hedonic and homeostatic food motivation are more robustly hypoactivated. Alterations in hedonic food motivation and cognitive control are likely to both be critically involved in the anorexigenic effects of oxytocin, as it has been shown that cognitive control and food reward sensitivity combined (with food reward sensitivity measured with the Power of Food Scale and cognitive control assessed by the ability to choose larger, delayed over smaller, immediate rewards) represent the best predictor of palatable food intake across the weight spectrum (Appelhans et al, 2011; Ely et al, 2015).

\section{FUNDING AND DISCLOSURE}

This study was supported by the Boston Nutrition Obesity Research Center/NIH Grant 5P30DK046200-20, Nutrition Obesity Research Center at Harvard/NIH Grant P30DK040561, and NIH Grant K23-MH092560. Dr Lawson is a member of the scientific advisory board of OXT Therapeutics and has a financial interest in the company. This company was not involved in any way in this research. The remaining authors declare no conflict of interest.

\section{ACKNOWLEDGMENTS}

We would like to thank our participants as well as the staff at the Massachusetts General Hospital Clinical Research Center and Athinoula A. Martinos Center for Biomedical Imaging.

\section{REFERENCES}

Anagnostou E, Soorya L, Chaplin W, Bartz J, Halpern D, Wasserman S et al (2012). Intranasal oxytocin versus placebo in the treatment of adults with autism spectrum disorders: a randomized controlled trial. Mol Autism 3: 16.

Appelhans BM, Woolf K, Pagoto SL, Schneider KL, Whited MC, Liebman R (2011). Inhibiting food reward: delay discounting, food reward sensitivity, and palatable food intake in overweight and obese women. Obesity 19: 2175-2182.

Arletti R, Benelli A, Bertolini A (1989). Influence of oxytocin on feeding behavior in the rat. Peptides 10: 89-93.

Blevins JE, Baskin DG (2015). Translational and therapeutic potential of oxytocin as an anti-obesity strategy: insights from rodents, nonhuman primates and humans. Physiol Behav 152(Pt B): $438-449$.

Blevins JE, Graham JL, Morton GJ, Bales KL, Schwartz MW, Baskin DG et al (2015). Chronic oxytocin administration inhibits food intake, increases energy expenditure, and produces weight loss in fructose-fed obese rhesus monkeys. Am J Physiol Regul Integr Comp Physiol 308: R431-R438.

Boccia ML, Petrusz P, Suzuki K, Marson L, Pedersen CA (2013). Immunohistochemical localization of oxytocin receptors in human brain. Neuroscience 253: 155-164.

Botvinick MM (2007). Conflict monitoring and decision making: reconciling two perspectives on anterior cingulate function. Cogn Affect Behav Neurosci 7: 356-366.

Calvo D, Galioto R, Gunstad J, Spitznagel MB (2014). Uncontrolled eating is associated with reduced executive functioning. Clin Obes 4: 172-179.

De Silva A, Salem V, Matthews PM, Dhillo WS (2012). The use of functional MRI to study appetite control in the CNS. Exp Diabet Res 2012: 764017.

Dimitropoulos A, Tkach J, Ho A, Kennedy J (2012). Greater corticolimbic activation to high-calorie food cues after eating in obese vs. normal-weight adults. Appetite 58: 303-312.

Domes G, Heinrichs M, Glascher J, Buchel C, Braus DF, Herpertz SC (2007). Oxytocin attenuates amygdala responses to emotional faces regardless of valence. Biol Psychiatry 62: 1187-1190.

Dreisbach G, Fischer R (2012). Conflicts as aversive signals. Brain Cogn 78: 94-98.

Ely AV, Howard J, Lowe MR (2015). Delayed discounting and hedonic hunger in the prediction of lab-based eating behavior. Eat Behav 19: 72-75.

Gao S, Becker B, Luo L, Geng Y, Zhao W, Yin Y et al (2016). Oxytocin, the peptide that bonds the sexes also divides them. Proc Natl Acad Sci USA 113: 7650-7654.

Gloy VL, Briel M, Bhatt DL, Kashyap SR, Schauer PR, Mingrone G et al (2013). Bariatric surgery versus non-surgical treatment for obesity: a systematic review and meta-analysis of randomised controlled trials. Br Med J 347: f5934.

Gregory R, Cheng H, Rupp HA, Sengelaub DR, Heiman JR (2015). Oxytocin increases VTA activation to infant and sexual stimuli in nulliparous and postpartum women. Horm Behav 69: 82-88.

Herisson FM, Waas JR, Fredriksson R, Schioth HB, Levine AS, Olszewski PK (2016). Oxytocin acting in the nucleus accumbens core decreases food intake. J Neuroendocrinol 28. (http:// onlinelibrary.wiley.com/doi/10.1111/jne.12381/abstract).

Holsen LM, Lawson EA, Blum J, Ko E, Makris N, Fazeli PK et al (2012). Food motivation circuitry hypoactivation related to hedonic and nonhedonic aspects of hunger and satiety in women 
with active anorexia nervosa and weight-restored women with anorexia nervosa. J Psychiatry Neurosci 37: 322-332.

LaBar KS, Gitelman DR, Parrish TB, Kim YH, Nobre AC, Mesulam MM (2001). Hunger selectively modulates corticolimbic activation to food stimuli in humans. Behav Neurosci 115: 493-500.

Lawson EA, Marengi DA, DeSanti RL, Holmes TM, Schoenfeld DA, Tolley CJ (2015). Oxytocin reduces caloric intake in men. Obesity 23: 950-956.

Loup F, Tribollet E, Dubois-Dauphin M, Dreifuss JJ (1991). Localization of high-affinity binding sites for oxytocin and vasopressin in the human brain. An autoradiographic study. Brain Res 555: 220-232.

Maldjian JA, Laurienti PJ, Kraft RA, Burdette JH (2003). An automated method for neuroanatomic and cytoarchitectonic atlasbased interrogation of fMRI data sets. NeuroImage 19: 1233-1239.

Mansouri FA, Tanaka K, Buckley MJ (2009). Conflict-induced behavioural adjustment: a clue to the executive functions of the prefrontal cortex. Nat Rev Neurosci 10: 141-152.

Melis MR, Melis T, Cocco C, Succu S, Sanna F, Pillolla G et al (2007). Oxytocin injected into the ventral tegmental area induces penile erection and increases extracellular dopamine in the nucleus accumbens and paraventricular nucleus of the hypothalamus of male rats. Eur J Neurosci 26: 1026-1035.

Mickey BJ, Heffernan J, Heisel C, Pecina M, Hsu DT, Zubieta JK et al (2016). Oxytocin modulates hemodynamic responses to monetary incentives in humans. Psychopharmacology 233: 3905-3919.

Miller EK, Cohen JD (2001). An integrative theory of prefrontal cortex function. Ann Rev Neurosci 24: 167-202.

Mullis K, Kay K, Williams DL (2013). Oxytocin action in the ventral tegmental area affects sucrose intake. Brain Res 1513: 85-91.

Nawijn L, van Zuiden M, Koch SB, Frijling JL, Veltman DJ, Olff M (2016). Intranasal oxytocin enhances neural processing of monetary reward and loss in post-traumatic stress disorder and traumatized controls. Psychoneuroendocrinology 66: 228-237.

Nederkoorn C, Smulders FT, Havermans RC, Roefs A, Jansen A (2006). Impulsivity in obese women. Appetite 47: 253-256.

Niendam TA, Laird AR, Ray KL, Dean YM, Glahn DC, Carter CS (2012). Meta-analytic evidence for a superordinate cognitive control network subserving diverse executive functions. Cogn Affect Behav Neurosci 12: 241-268.

Ogden CL, Carroll MD, Kit BK, Flegal KM (2014). Prevalence of childhood and adult obesity in the United States, 2011-2012. J Am Med Assoc 311: 806-814.

Olson BR, Drutarosky MD, Chow MS, Hruby VJ, Stricker EM, Verbalis JG (1991). Oxytocin and an oxytocin agonist administered centrally decrease food intake in rats. Peptides 12: 113-118.

Ott V, Finlayson G, Lehnert H, Heitmann B, Heinrichs M, Born J et al (2013). Oxytocin reduces reward-driven food intake in humans. Diabetes 62: 3418-3425.

Paffenbarger RS Jr, Blair SN, Lee IM, Hyde RT (1993). Measurement of physical activity to assess health effects in free-living populations. Med Sci Sports Exerc 25: 60-70.
Parent A (1996). Carpenter's Human Neuroanatomy9th edn. Williams \& Wilkins: Baltimore, MD, USA.

Paxinos G, Huang X-F (1995). Atlas of the Human Brainstem. Academic Press: San Diego, CA, USA.

Riem MM, van IMH, Tops M, Boksem MA, Rombouts SA, Bakermans-Kranenburg MJ (2012). No laughing matter: intranasal oxytocin administration changes functional brain connectivity during exposure to infant laughter. Neuropsychopharmacology 37: 1257-1266.

Saper CB, Chou TC, Elmquist JK (2002). The need to feed: homeostatic and hedonic control of eating. Neuron 36: 199-211.

Sellaro R, Colzato LS (2017). High body mass index is associated with impaired cognitive control. Appetite 113: 301-309.

Skuse DH, Gallagher L (2009). Dopaminergic-neuropeptide interactions in the social brain. Trends Cogn Sci 13: 27-35.

Sofroniew MV (1983). Morphology of vasopressin and oxytocin neurones and their central and vascular projections. Prog Brain Res 60: 101-114.

Spielberger CD, Gorsuch RL, Lushene R, Vagg PR, Jacobs GA (1983). Manual for the Stait-Trait Anxiety Scale. Consulting Psychologists: Palo Alto, CA, USA.

Stojek MMK, MacKillop J (2017). Relative reinforcing value of food and delayed reward discounting in obesity and disordered eating: a systematic review. Clin Psychol Rev 55: 1-11.

Striepens N, Schroter F, Stoffel-Wagner B, Maier W, Hurlemann R, Scheele D (2016). Oxytocin enhances cognitive control of food craving in women. Hum Brain Mapp 37: 4276-4285.

Stunkard AJ, Messick S (1985). The three-factor eating questionnaire to measure dietary restraint, disinhibition and hunger. $J$ Psychosom Res 29: 71-83.

Thienel M, Fritsche A, Heinrichs M, Peter A, Ewers M, Lehnert $\mathrm{H}$ et al (2016). Oxytocin's inhibitory effect on food intake is stronger in obese than normal-weight men. Int J Obes 40; 1707-1714.

van der Klaauw AA, Ziauddeen H, Keogh JM, Henning E, Dachi S, Fletcher PC et al (2017). Oxytocin administration suppresses hypothalamic activation in response to visual food cues. Sci Rep 7: 4266.

Whalen PJ, Bush G, McNally RJ, Wilhelm S, McInerney SC, Jenike MA et al (1998). The emotional counting Stroop paradigm: a functional magnetic resonance imaging probe of the anterior cingulate affective division. Biol Psychiatry 44: 1219-1228.

Wigton R, Radua J, Allen P, Averbeck B, Meyer-Lindenberg A, McGuire $\mathrm{P}$ et al (2015). Neurophysiological effects of acute oxytocin administration: systematic review and meta-analysis of placebo-controlled imaging studies. J Psychiatry Neurosci 40: E1-22.

Yanovski SZ, Yanovski JA (2014). Long-term drug treatment for obesity: a systematic and clinical review. J Am Med Assoc 311: 74-86.

Zhang H, Wu C, Chen Q, Chen X, Xu Z, Wu J et al (2013). Treatment of obesity and diabetes using oxytocin or analogs in patients and mouse models. PLoS ONE 8: e61477. 